\title{
Living with primary ciliary dyskinesia
}

\section{The patient and healthcare professional perspective}

\section{Patient perspective}

Fiona Copeland: the patient's mother

Gregor had symptoms of primary ciliary dyskinesia (PCD) from birth. He was born with a cough and a cold and ended up being admitted into a special care unit after 24 hours with pneumonia. It wasn't until he was 4 years old that he received the diagnosis of PCD.

Gregor was generally unwell when he was little. He received a lot of treatment for what his doctors thought was asthma including several courses of steroids. This treatment caused his bones to be weak and when he had an injury playing sport it took a long time to heal. If he had received the correct diagnosis sooner we might have avoided the overuse of steroid treatment. He also experienced hearing issues when he was younger because of PCD, which meant he was very late in speaking and had to see a speech therapist.

We have two children with PCD, which has been hard work. I have had to give up working full time to look after them. Now Gregor is 16 years old he manages his condition himself a lot more, but we still have to take him to hospital appointments, get sputum samples delivered to the hospital for testing when he isn't well and arrange to get his medication from the GP/pharmacist. We have also been trained to administer intravenous antibiotics at home when they are needed.
We have tried not to wrap the boys in cotton wool and encourage them to lead active lives and eat healthy diets. Missing a lot of school has been difficult for Gregor and he had big gaps in his primary education.

We can contact respiratory nurse specialists at our local and specialist hospital at any time if we need an urgent appointment. You can live a "normal life" if you have PCD but it is hard work keeping the children well. Physiotherapy is really important to do twice a day and it is not negotiable in our house.... Every morning and every night even on birthdays and Christmas!

\section{Gregor Copeland: the patient}

The main symptoms I experience as part of my condition are a runny nose, coughing, wheezing and hearing problems. I was generally unwell as a child and I think that if I had been diagnosed sooner, it may have reduced the amount of lung damage I experienced before and it may also have prevented my delayed speech.

My healthcare professional has provided lots of advice about managing my symptoms. I do physiotherapy twice a day using nebulised hypertonic saline before each session. This takes about 20 minutes each time. I take inhalers to stop me wheezing and tablets to stop me suffering from reflux (they stop me feeling sick and getting heartburn). I sometimes have to take antibiotics too. I also 
have to regularly squirt warm salty water up my nose to help clear my nose and sinuses. I also try to do exercise two or three times a week, which helps me clear my chest.

My daily life is spent doing all the things the doctors tell me to do. I know that if I don't do these things, particularly the physiotherapy, then I feel much worse. When I was younger, I had a lot of time off school and I used to get lots of chest infections that required intravenous antibiotics. This would mean I could be in hospital for up to 3 weeks. Nowadays, if I get an infection I get a temperature and my cough gets worse.

Because I had so many intravenous antibiotics when I was younger, my veins were difficult to get lines into. I had to have a portacath fitted. My mum now has to look after this every month by injecting medicines into it for me.

I regularly visit my local hospital and a specialist hospital in the UK. I go to see them about every 6 months or more regularly if I am poorly. I find these meetings useful as they provide me with a general health check, so I know how I am doing.

My main piece of advice for someone newly diagnosed with PCD would be to do their physiotherapy every day. If you are finding that you can't fit it in every morning and night then ask your physiotherapist about the best way to do it so it doesn't interrupt your normal life.

\section{The healthcare professional's perspective: Claire Hogg}

Claire Hogg is a paediatric respiratory consultant at the Royal Brompton Hospital (London, UK).

I have been Gregor's consultant for 8 years now, but I was also involved in his care for several years before that. Gregor, and his older brother, were referred from their local hospital with suspected PCD because of their clinical symptoms of persistent rhinitis and chronic wet cough. Gregor's brother Euan also had evidence of bronchiectasis at this stage, and so the boys had had other causes of this condition excluded, such as cystic fibrosis. At the time of their diagnosis we had limited access to a reliable screening test, and our diagnostic service, like elsewhere in the UK, was undertaken through our research facilities.

As we didn't have a suitable screening test at the time of their referral, both boys had a brush biopsy taken from their noses. This gives us a sample of ciliated epithelium to analyse, and will tell us if the ciliary function is abnormal throughout the airway. Initially the sample is looked at using a light microscope to assess ciliary beat pattern and frequency. In PCD the ciliary beat pattern is very abnormal, ranging from having static cilia to dyskinetic or chaotic beat patterns with ineffective mucociliary clearance.

The ciliary beat pattern is the most consistently abnormal test in cases of PCD; however, it is also very important to look for the definitive ultrastructural abnormality within the ciliary axoneme. The axoneme is made up of protein components including microtubules, dynein arms, radial spokes and nexin links, amongst others, that allow cilial integrity and movement. By processing the nasal sample the individual cilia can be fixed and cut to allow high definition images to be interpreted using a transmission electron microscope (TEM). $85 \%$ of PCD cases have an identifiable ultrastructural defect using TEM. The majority involve absence of the outer dynein arms (ODA), or a combination of missing ODA and inner dynein arms (IDA). Gregor cilia were static when viewed using light microscopy, and he had the diagnosis confirmed when TEM demonstrated absence of the ODA.

Diagnosing PCD is invasive, time consuming and expensive. The tests described above take, at best, 4-6 weeks to complete, but often a sample needs to be repeated if the initial brush biopsy is unhealthy or insufficient. Since Gregor was diagnosed, we now have same day screening using a test called nasal nitric oxide. This has revolutionised our diagnostic service, often allowing us to make accurate assumptions about which patients need to go on to have the invasive diagnostic tests and which patients need to start directed treatment at the point of referral whilst we await the more definitive diagnostic test results.

Research is currently focused on gene discovery studies, which have led to the identification of 27 known disease-causing genes to date, a gene panel that can now be clinically applied to patient groups for gene identification. Genotyping currently identifies around $60 \%$ of patients with PCD and so it is not a stand-alone diagnostic test.

Radio-labelled immunofluorescent antibody staining techniques are also of great 
interest. This technique utilises labelled antibodies that specifically bind to and label the main axonemal proteins such as ODA, IDA, radial spokes and nexin links. Immunofluorescent techniques have the advantage of requiring less biopsy material, having a rapid turnaround within 24 hours, and are relatively cheap. Immunofluorescent techniques remain a research test, but as research in this area continues to show huge promise, it is likely to become part of the diagnostic pathway in the near future.

Despite these advances there is still a subgroup of patients who have a strong clinical phenotype, positive screening test and abnormal ciliary beat pattern, but for whom we cannot confirm the diagnosis using TEM, genetics or immunofluorescent techniques.

As PCD is a multisystem disease, it requires a multi-specialist approach and should, wherever possible, not be managed alongside other forms of chronic lung disease where the needs are often different. Specialist PCD clinics with a team including a chest physician, an ear, nose and throat surgeon, an audiologist, a physiotherapist and a clinical nurse specialist should be the norm. Additional access to psychology, dietetics, cardiology and fertility experts in adult clinics is also key.

Every visit to the clinic or ward involves seeing the physiotherapist, reinforcing techniques and adapting techniques to suit age, lifestyle and state of well-being to try and improve compliance. A low threshold for treating infection is essential. Retained secretions lead to increased risk of infection, and infected secretions result in release of damaging inflammatory mediators that can cause airway damage and progressive lung disease.

Patients suffer persistent rhinosinusitis, glue ear, hearing loss and difficulties with speech development. Unmanaged, this can lead to delayed milestones and poor educational achievements with life-long consequences for future education and job prospects. Specialist management and monitoring of these problems is essential to prevent this cycle of events, and we provide outreach to home and school to overcome the issues that might arise when parents and teachers are unaware of the potential impact of PCD on a child's development.

Education about the importance of lung health is a key feature of our management approach. People with PCD often feel quite well and as a result they ignore their treatments preferring to live with the daily symptoms that they are quite used to. Convincing them to do time consuming treatments is the biggest task we face, and we use our multidisciplinary team approach to address this.

When a person is first diagnosed with PCD, it is often as a child and we discuss the condition with their parents. The aim is to develop an understanding of how maintaining good lung health is essential to leading a long and normal life. It is then our responsibility to engage the child in understanding about their lung health as they grow and start to take responsibility for their own treatments.

It is also important for the healthcare professional to promote general lung health protection measures such as vaccination against pneumonia and influenza and antismoking advice; occupational exposure to both infection and industrial pollutants also plays an important role.

It is key that we raise awareness about the cardinal signs of PCD among clinicians. The common presenting signs of PCD are all common childhood illnesses and so PCD is often not considered. However, the key features that should prompt a referral to a specialist centre include: rhinitis from day $1-2$ of life, a history of unexplained respiratory distress/pneumonia in a new-born term infant, daily wet cough from the early weeks of life that is persistent regardless of infection status, presence of situs inversus (mirror image arrangement of heart and other organs), persistent glue ear, moderate-tosevere hearing loss, or a persistent discharge from the ears following perforation or insertion of grommets. 